EPJ Web of Conferences 92,02071 (2015)

DOI: $10.1051 /$ epjconf/ 20159202071

C) Owned by the authors, published by EDP Sciences, 2015

\title{
Molecular simulation of water vapor-liquid phase interfaces using TIP4P/2005 model
}

\author{
Barbora Planková ${ }^{1,2, a}$, Václav Vinš ${ }^{1}$, Jan Hrubý$^{1}$, Michal Duška $^{1}$, Tomáš Němec ${ }^{1}$, and David Celný ${ }^{2}$ \\ 1 Institute of Thermomechanics, Academy of Sciences of the Czech Republic, v. v. i., Dolejškova 1402/5, Prague, Czech \\ Republic \\ 2 Faculty of Nuclear Sciences and Physical Engineering, Czech Technical University in Prague, Břehová 78/7, Prague, \\ Czech Republic
}

\begin{abstract}
Molecular dynamics simulations for water were run using the TIP4P/2005 model for temperatures ranging from $250 \mathrm{~K}$ to $600 \mathrm{~K}$. The density profile, the surface tension and the thickness of the phase interface were calculated as preliminary results. The surface tension values matched nicely with the IAPWS correlation over wide range of temperatures. As a partial result, DL_POLY Classis was successfully used for tests of the new computing cluster in our institute.
\end{abstract}

\section{Introduction}

Water is perhaps the most studied substance in the world due to its importance in daily life, industry or physical, chemical or biological processes. Due to its many anomalies and non-standard behavior it is however very hard to model. The non-trivial phenomena are caused by its polar character and consequently by its formation of hydrogen bonds.

As for numerical simulations, water has been studied extensively over past decades with the pioneering works by Barker and Watts [1] and Rahman and Stillinger [2]. Despite its compexity, simple models of water are widely used, because they work surprisingly well. This moreover allows computation of more extensive systems consisting of thousands of molecules. Many simple models are developed, but as the best of them are considered SPC/E model and TIP4P/2005 model [3,4].

Motivation of our work is to shed light on the discrepancies between experiments and simulations, e.g. the second inflection point of water [5], to reproduce measurements of the surface tension of supercooled water as well as to enhance our theoretical work concerning nucleation rates predictions [6] and capillary waves modeling [7].

In this paper we publish preliminary results of part of our newly formed simulation group. Primary objective was to get the software executing the simulation procedure working in our new cluster in the direction of our interests. We used DL_POLY Classic program on $4 \times 24$ Intel(R) Xeon(R) CPU E5645 @ 2.40GHz CPUs, debugging and early computations were executed on 4 AMD Phenom (tm) 9600 Quad-Core GPUs.

We simulated a water slab for various temperatures from $250 \mathrm{~K}$ towards the critical point (which was not exceeded) stopping at $T=600 \mathrm{~K}$. To have a relevant reference point, we followed specifications of papers [8] and [9].

\footnotetext{
a e-mail: barbora.plankova@gmail.com
}

Table 1. Simulation parameters of TIP4P/2005 water molecule atoms: oxygen $\mathrm{O}$, hydrogen $\mathrm{H}$, mass-less charge $\mathrm{M}$. $m$ is molar mass, $q$ charge in units of elementary charge, $\epsilon$ and $\sigma$ are Lennard-Jones parameters.

\begin{tabular}{c|cccc} 
atom & $m(\mathrm{~g} / \mathrm{mol})$ & $q(\mathrm{e})$ & $\epsilon(\mathrm{kJ} / \mathrm{mol})$ & $\sigma(\AA)$ \\
\hline $\mathrm{O}$ & 15.99940 & 0.000 & 0.77490 & 3.1589 \\
$\mathrm{H}$ & 1.00794 & 0.5564 & - & - \\
$\mathrm{M}$ & 0.00000 & -1.1128 & - & -
\end{tabular}

\section{TIP4P/2005 model}

Although water is flexible and polarizable, simple rigid non-polarizable models are used in most of simulations. The reason is that some of them represent experimental data very well, which allows one to model larger or longerrunning systems. All the simple models from SPC or TIPXP families have in common that they locate the positive charge on the hydrogen atoms and a Lennard-Jones interaction site on the position of the oxygen. They however differ in bond geometry (angle of $\mathrm{H}-\mathrm{O}-\mathrm{H}$ bonds), charge distribution or their target properties. Three-site models TIP3P [10] and SPC [11] were developed in 1980s. An SPC modification, SPC/E model [12], is very much used nowadays. SPC and SPC/E models differ in a way that SPC/E includes some empirical corrections to a polarization energy. This model became very successful and reproduces data very well.

To improve the electrostatic distribution within the water molecule, an auxilliary atom $\mathrm{M}$ was introduced in TIP4P [10] model. This atom carries the negative charge, is massless and close to oxygen atom $(0.15 \AA)$. Probably the best model is TIP4P/2005, proposed by Vega and Abascal [13] in 2005. They tried to combine good phase diagram of TIP4P with target properies of SPC/E improving the melting point. It has been shown $[3,4]$ that TIP4P/2005 behavior is even better than that of SPC/E. Therefore, in this work we use the TIP4P/2005 model. Another extension TIP5P was proposed by Mahoney et al. [14] to carry the 
negative charge on two auxiliary atoms. However, the performance is not better than of TIP4P/2005.

TIP4P/2005 model includes Lennard-Jones interactions between oxygen atoms only; hydrogens have negligible mass compared to them, which makes the simulation easier. Other interaction is electrostatic which occurs between hydrogen $\mathrm{H}$ and charge $\mathrm{M}$ atoms. TIP4P/2005 parameters are listed in table 1.

\section{Simulation methods}

On our new cluster we installed the DL_POLY Classic (former DL_POLY 2) and DL_POLY 4 programs. We performed computations using both, but then we decided to use DL_POLY Classic on this problem. The reason was that DL_POLY 4 is designed for larger systems and its usage was less efficient.

We performed a number of molecular dynamics simulations within NVT (canonical) ensemble, i.e. number of particles, volume and temperatures were fixed. For an initial configuration of molecules, we used phase centered cubic mesh where four molecules are put to a corners of one cube, these cubes are put next to each other with a blank space of a size of one cube. Therefore, number of molecules $N$ is restricted to numbers $4 \times N_{c}^{3}$, where $N_{c}$ is a positive integer (see table 2 ). We chose the number of molecules of 1372 which is quite close to 1024 molecules considered by Sakamaki et al. [8].

The simulation was performed as follows: first, a liquid cubic box was run for $50 \mathrm{ps}$, then the $z$-size of the box was expanded to approximately $3 \times$ the original proportion and run for $10 \mathrm{~ns}$ to provide reliable data for surface tension determination. Sizes of the box were calculated depending on the NIST [15] values of the water saturated liquid density for particular temperature. For supercooled region, a constant box size corresponding to $300 \mathrm{~K}$ system was used. Periodic boundary conditions were used in all directions. This imposed a small problem in extension of the system in z-direction - molecule can be on boundaries of the simulation box, therefore, simple extension of the axis would "break" the molecule. Between the two simulations, a Fortran code was run to rotate the molecules so that none of them is on the boundary of the $z$-direction. Timestep of the simulation was chosen as $2 \mathrm{fs}$ (same as in [8]) with velocity Verlet integrator. To maintain constant temperature, we used the Nosé-Hoover thermostat with relaxation constant 100 fs. Cutoffs of Lennard-Jones interactions and van der Waals forces were set to $14.5 \AA$, close to the value in [8]. For electrostatic interactions, direct Ewald method was used, with automatic parameter optimization constant set to $10^{-5}$. This value gave a convergence parameter $0.192 \AA^{-1}$ and k-vector indeces $6 \times 6 \times 6$ for a cubic box, $6 \times 6 \times 18$ for slab geometry. For higher temperatures, the values got up to $7 \times 7 \times 7$ and $7 \times 7 \times 29$ respectively. Density was computed as a histogram in $z$-direction in every step and averaging through the time. Examples of the density profiles converted to $\mathrm{g} / \mathrm{cm}^{3}$ for 300 and $500 \mathrm{~K}$ can be seen in figure 1 depicted by the solid lines. Snapshots of the simulations for two temperatures are shown in figure 2.

The density profiles were divided to two halves approximately in the centre of the simulation box. One half of the density profile was subsequently fitted to a hyperbolic tan-
Table 2. Number of molecules $N$ permitted by the phase centered cubic mesh algorithm of the water molecules initial configuration. $N_{c}$ is arbitrary positive integer.

\begin{tabular}{|c|c|}
\hline$N_{c}$ & $N$ \\
\hline 3 & 108 \\
4 & 256 \\
5 & 500 \\
6 & 864 \\
7 & 1372 \\
8 & 2048 \\
9 & 2916 \\
10 & 4000 \\
\hline
\end{tabular}

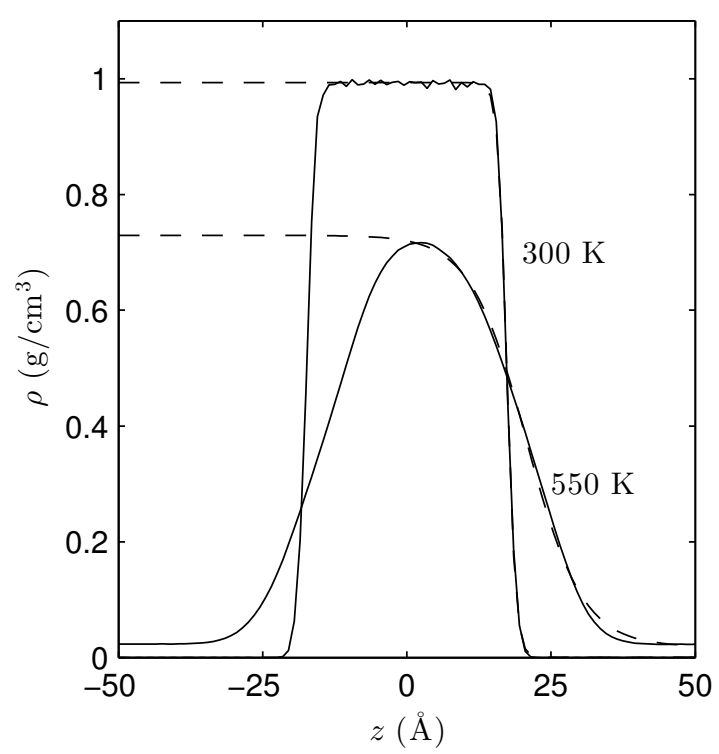

Fig. 1. Density profiles $\rho$ as functions of $z$-coordinate for systems having temperatures $T=300 \mathrm{~K}$ and $500 \mathrm{~K}$. Solid lines are timeaveraged profiles obtained from the simulations, dashed lines are fits of the right-hand profiles (liquid - vapor) to the hyperbolic tangent profile, equation (1).

gent density profile model,

$$
\rho(z)=\frac{\rho_{\mathrm{L}}+\rho_{\mathrm{V}}}{2}-\frac{\rho_{\mathrm{L}}-\rho_{\mathrm{V}}}{2} \tanh \left(\frac{z-z_{0}}{d}\right),
$$

where $\rho_{\mathrm{L}}$ and $\rho_{\mathrm{V}}$ are fitted bulk densities, $z_{0}$ is the position of the Gibbs dividing surface of the interface, $d$ is the parameter for the thickness of the interface. For $z$ and $\rho$ we used the simulation data to be fitted. The results of the fit are depicted as the dashed lines in figure 1 . The fitted values were used by evaluating the surface tension $\gamma$ in the following manner:

$$
\begin{aligned}
\gamma & =\frac{L_{\mathrm{z}}}{2}\left(P_{\mathrm{zz}}-\frac{P_{\mathrm{xx}}+P_{\mathrm{yy}}}{2}\right)+12 \pi \epsilon \sigma^{6}\left(\rho_{\mathrm{L}}-\rho_{\mathrm{V}}\right)^{2} \\
& \times \int_{0}^{1} \int_{r_{\mathrm{c}}}^{\infty} \operatorname{coth}\left(\frac{r s}{d}\right) \frac{3 s^{3}-s}{r^{3}} \mathrm{~d} r \mathrm{~d} s .
\end{aligned}
$$

In equation (2), $L_{\mathrm{z}}$ denotes the box size in $z$ direction, $P_{\mathrm{ii}}$ is the $i i$-th component of the pressure tensor, $\epsilon$ and $\sigma$ are the Lennard-Jones parameters for oxygen atom, and $r_{\mathrm{c}}$ is the cutoff for the Lennard-Jones potential. Their values as well as other model parameters are summarized in table 1. 
a)

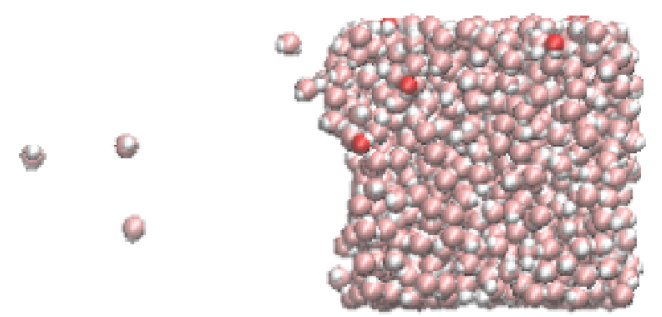

b)
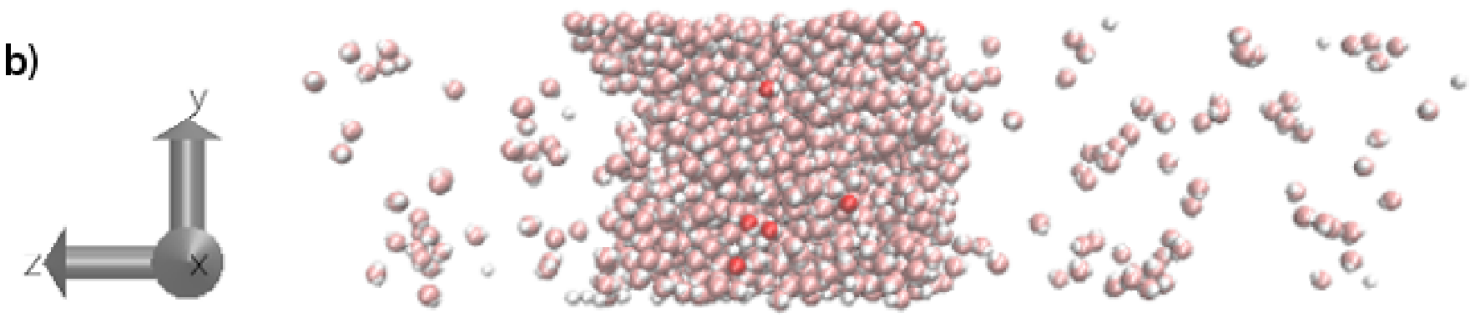

Fig. 2. Snapshots of two configurations during the simulations for two temperatures, a) $T=300 \mathrm{~K}$ and b) $550 \mathrm{~K}$. Liquid cubes are in the middle, vapor is on the left and right side (not so apparent for lower temperature).

Second term in equation (2) corresponds to the LennardJones tail correction [16].

\section{Results}

Insipired by the work of Sakamaki et al. [8], we performed molecular dynamics simulations of a water slab enclosed by the vapor for temperatures $T=250 \mathrm{~K}, 270 \mathrm{~K}, 275 \mathrm{~K}$, $300 \mathrm{~K}, 350 \mathrm{~K}, 400 \mathrm{~K}, 450 \mathrm{~K}, 500 \mathrm{~K}, 550 \mathrm{~K}$, and $600 \mathrm{~K}$. Dimensions of the box varied from $34.53 \AA$ corresponding to a density of liquid water at $300 \mathrm{~K}$ which was used also for systems at temperatures below $300 \mathrm{~K}$ up to $39.83 \AA$ for $600 \mathrm{~K}$. We decided to vary the simulation box sizes, since it corresponded to reality better than box with constant sizes. Moreover for $600 \mathrm{~K}$, small box forced the almost incompressible liquid water to too low volume which caused an "explosion" of the liquid cube, shifted the slab and made the system unnecessarily non-equilibrium at start of the slab simulation.

Given by the parameters stated in previous section, computations ran approximately 3 days if running on all 24 CPUs. Melting temperature for TIP4P $/ 2005$ is $T_{\mathrm{m}}=249 \mathrm{~K}$, therefore even for lowest temperature of $250 \mathrm{~K}$ we did not encounter any of the liquid water during the simulation.

Figure 1 shows density $\rho$ varying with the $z$ coordinate averaged through the simulation. The structure of the simulation box can be seen: there is vapor on the left and right side of the $z$ axis, while the liquid phase is allocated in the centre of the box. For the purposes of parameters fitting, only the right half of the profile was necessary. As the temperature increased, the bulk liquid density decreased and bulk vapor density increased.

Figure 2 shows an example of two configurations for two temperatures $(300 \mathrm{~K}, 550 \mathrm{~K})$. The liquid phase persisted in the centre of the simulation box, while the vapor phase gradually expanded into the vacuum space after the box got stretched in the $z$-direction. As can be seen at low temperatures, the molecule escaping from the liquid phase

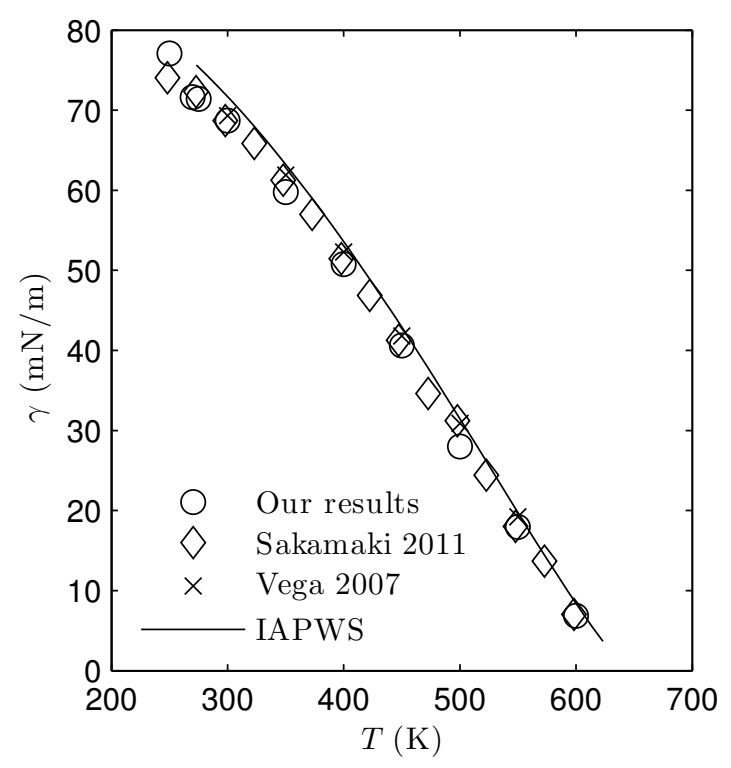

Fig. 3. Surface tension $\gamma$ as a function of temperature $T$ as predicted by this work, computed using equation (2) (circles), compared to the IAPWS values $[17,15]$ (solid line).

into the vapor phase was rather rare event. On the other hand at the elevated temperatures, the vapor phase got significantly denser.

Surface tension computed using equation (2) is shown in figure 3 as circles, the IAPWS values $[17,15]$ are shown as a solid line. Simulated values nicely describe the reference data.

Figure 4 shows the thickness of the profile $t$, known also as the "90-10" thickness defined as $t=2.1972 d$, where $d$ is the thickness parameter obtained from equation (1). An exponential trend can be observed which agrees with the temperature scaling along the critical point (critical scaling theory) $[18,19]$. 


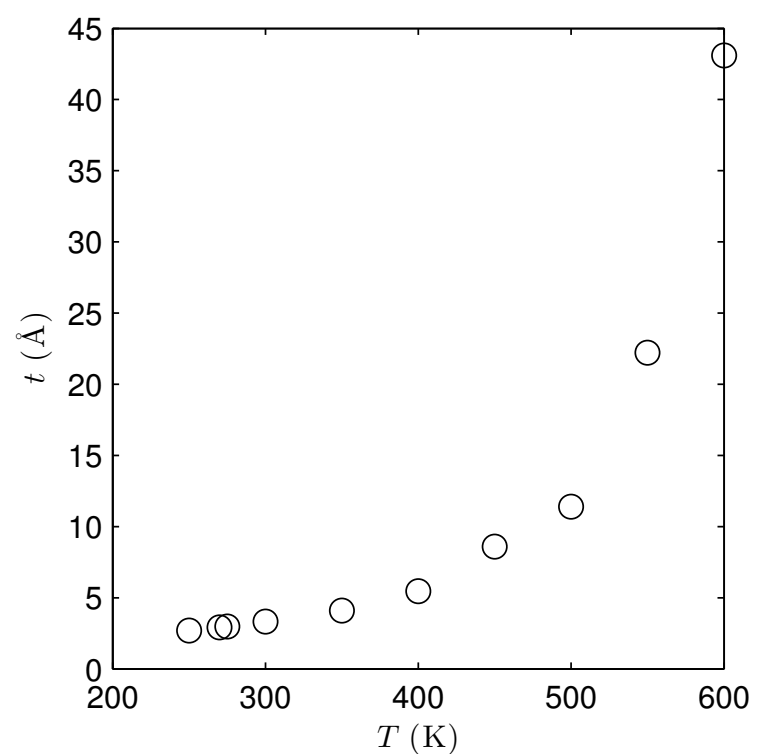

Fig. 4. Thickness of the profile $t$ as a function of temperature $T$ predicted in this work using the tangent hyperbolic fit of parameter $d$ and reevaluation $t=2.1972 d$.

\section{Conclusions}

In this work we performed molecular dynamics simulations for water for various temperatures ranging from $250 \mathrm{~K}$ to $600 \mathrm{~K}$. As a water model we used TIP4P/2005 which is probably the best rigid non-polarizable water model available at the moment. We computed surface tension as well as the interface thickness using the hyperbolic tangent fit as preliminary results.

Main goal of this paper was to test our new computing cluster on molecular simulations and to investigate the performance of the DL_POLY Classic software. Surface tension data for water fitted nicely to the IAPWS tabulated values $[17,15]$. In future, we would like to perform more simulations in the supercooled region of liquid water using the TIP4P/2005 and the SPC/E models to compare simulated results with our recent experiments. [5].

Also, we would like to model the so-called capillary waves contribution to the surface tension, i.e. to simulate, how the thermal motion of molecules affects (lowers) the surface tension for planar phase interface. New molecular simulations would support our theoretical work [6,7,2022].

\section{Acknowledgement}

We are very grateful to dr. Martin Lísal (ICPF AS CR) who taught us the basics of numerical simulations and provided us with valuable advices concerning the simulation parameters. The study has been supported by grant No. 7F14466 of Czech-Norwegian Research Programme CZ09, Czech Science Foundation grant No. P101/11/1593, and institutional support RVO:61388998.

\section{References}

1. J. Barker, R. Watts, Chemical Physics Letters 3, 144 (1969)
2. A. Rahman, F.H. Stillinger, The Journal of Chemical Physics 55, 3336 (1971)

3. C. Vega, J.L. Abascal, M. Conde, J. Aragones, Faraday discussions 141, 251 (2009)

4. C. Vega, J.L. Abascal, Physical Chemistry Chemical Physics 13, 19663 (2011)

5. J. Hrubý, V. Vinš, R. Mareš, J. Hykl, J. Kalová, J. Phys. Chem. Lett. 5, 425 (2014)

6. B. Planková, J. Hrubý, V. Vinš, Prediction of the homogeneous droplet nucleation by the density gradient theory and PC-SAFT equation of state, in Nucl. and Atmos. Aerosols: 19th Int. Conf. (2013)

7. J. Hrubý, B. Planková, V. Vinš, Corrections to the classical work of formation of critical clusters, in Nucl. and Atmos. Aerosols: 19th Int. Conf. (2013)

8. R. Sakamaki, A.K. Sum, T. Narumi, K. Yasuoka, J. Chem. Phys. 134 (2011)

9. C. Vega, E. De Miguel, J. Chem. Phys. 126, 154707 (2007)

10. W.L. Jorgensen, J. Chandrasekhar, J.D. Madura, R.W. Impey, M.L. Klein, The Journal of chemical physics 79, 926 (1983)

11. H.J. Berendsen, J.P.M. Postma, W.F. van Gunsteren, A. DiNola, J. Haak, The Journal of chemical physics 81, 3684 (1984)

12. H. Berendsen, J. Grigera, T. Straatsma, Journal of Physical Chemistry 91, 6269 (1987)

13. J.L. Abascal, C. Vega, The Journal of chemical physics 123, 234505 (2005)

14. M.W. Mahoney, W.L. Jorgensen, The Journal of Chemical Physics 112, 8910 (2000)

15. E. Lemmon, M. McLinden, D. Friend, in NIST Chemistry WebBook, NIST Standard Reference Database Number 69, edited by P. Linstrom, W. Mallard (National Institute of Standards and Technology, Gaithersburg MD, 2014 (retrieved September 10)), http://webbook.nist.gov

16. G.A. Chapela, G. Saville, S.M. Thompson, J.S. Rowlinson, J. Chem. Soc., Faraday Trans. II 73, 1133 (1977)

17. IAPWS release on surface tension of ordinary water substance (1994), http: //www . iapws . org/

18. S. Fisk, B. Widom, J. Chem. Phys. 50, 3219 (1969)

19. M.E. Fisher, Reviews of Modern Physics 70, 653 (1998)

20. V. Vinš, J. Hrubý, B. Planková, Proceedings of the international conference, in Experimental Fluid Mechanics 2010 (Liberec, 2010)

21. B. Planková, J. Hrubý, V. Vinš, Homogeneous droplet nucleation modeled using the gradient theory combined with the PC-SAFT equation of state, in EPJ Web of Conferences (EDP Sciences, 2013), Vol. 45, p. 01076

22. V. Vinš, J. Hrubý, B. Planková, International Journal of Thermophysics 34, 792 (2013) 